\title{
Low temperature precipitation kinetics of niobium nitride platelets in $\mathrm{Fe}$
}

\author{
A. Deschamps ${ }^{\text {a,* }}$, F. Danoix ${ }^{\text {b }}$, F. De Geuser ${ }^{\text {a }}$, T. Epicier ${ }^{c}$, H. Leitner $^{\text {d }}$, M. Perez ${ }^{\mathrm{c}}$ \\ a SIMAP, Grenoble INP - CNRS - UJF, BP 75, 38402 St Martin d'Hères Cedex, France

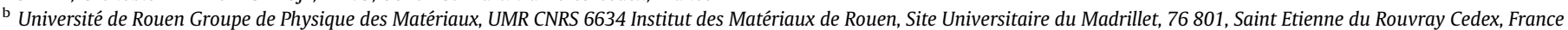 \\ c Université de Lyon, INSA Lyon, MATEIS, UMR CNRS 5510, 7, avenue Jean-Capelle, 69621 Villeurbanne Cedex, France \\ d Christian Doppler Laboratory Early Stages of Precipitation, Montanuniversität Leoben, Franz-Josef-Straße 18, A-8700 Leoben, Austria
}

\begin{abstract}
Single plane platelets of niobium nitride have been observed to form in a Fe-Nb-N alloy during ageing at $600{ }^{\circ} \mathrm{C}$, using High Resolution Electron Microscopy, Field Ion Microscopy and Atom Probe Tomography. Small-angle neutron scattering has been used to investigate the kinetics of formation of these platelets. They are shown to nucleate in less than $5 \mathrm{~min}$ at this ageing temperature, and subsequently to grow in-plane to a size of about $10 \mathrm{~nm}$ without experiencing any change in thickness.
\end{abstract}

Keywords: Precipitation, Steels, Small-angle neutron scattering Niobium nitride

\section{Introduction}

Precipitation in microalloyed steels is based on the combination of several metallic elements ( $\mathrm{Nb}, \mathrm{Ti}$, and $\mathrm{V}$ ) with carbon or nitrogen. Particularly, $\mathrm{Nb}$ is known to form carbonitrides of $\mathrm{NaCl}$ structure, where carbon and nitrogen substitute on the same lattice sites [1-4]. $\mathrm{Nb}-(\mathrm{C}, \mathrm{N})$ precipitates are widely used both for grain size control at high temperature in the austenitic phase and for strengthening at lower temperature in the ferritic phase [5-7].

Precipitation of $\mathrm{Nb}$ carbides or carbonitrides is well documented at temperatures corresponding to situations relevant to coiling of rolled steels (typically at and above $650{ }^{\circ} \mathrm{C}$ ). At lower temperature, niobium nitrides have received recently a renewed interest $[1,2,8]$ because a precursor phase forming at low temperature from the solid solution has been identified as being single plane $\mathrm{NbN}$ platelets, very similar to GP zones found e.g. in Aluminium alloys, and similar in shape to the early precipitates formed when nitriding steels [9-13]. Subsequently to these observations, their stability has been recently investigated by ab initio calculations [14]. However, until now no evaluation of the kinetics of precipitation of this precursor phase has been reported. It is not known how the very fine morphology of these platelets evolves with ageing time, or the relationship between the precipitation of these platelets and the hardness of the material.

In the present paper we report the precipitation kinetics of $\mathrm{NbN}$ platelets in a model Fe-Nb-N steel. The morphology of these platelets is identified by High Resolution Electron Microscopy (HREM), Field

\footnotetext{
* Corresponding author. Fax: + 33476826644 .

E-mail address: alexis.deschamps@grenoble-inp.fr (A. Deschamps).
}

Ion Microscopy (FIM) and Atom Probe Tomography (APT). The precipitation kinetics is then evaluated using Small-Angle Neutron Scattering (SANS) for various ageing times at $600{ }^{\circ} \mathrm{C}$. The measured kinetics is finally compared to hardness measurements.

\section{Material and experimental methods}

The investigated high purity Fe alloy contains 0.075 at\% N and 0.05 at\% $\mathrm{Nb}$. It contains less than $10 \mathrm{wt}$ ppm of other elements like C, S or O. After hot rolling at $1150{ }^{\circ} \mathrm{C}$, it was solution treated for $45 \mathrm{~min}$ at $1250{ }^{\circ} \mathrm{C}$, which resulted in a full solid solution, and then quenched in cold water. It was then heat treated in quartz tubes for different ageing times at $600{ }^{\circ} \mathrm{C}$.

Transmission Electron Microscopy (TEM) imaging was performed in a FEI-Titan microscope operated at $300 \mathrm{kV}$ and equipped with a Cs-corrector for imaging. Thin foils were prepared by conventional electropolishing ( $4 \%$ Nital solution) followed by ion-beam thinning.

Specimens for Field Ion Microscopy (FIM) and Atom Probe Tomography (APT) were prepared using the standard two-stage method. FIM investigations were conducted at temperature of $80 \mathrm{~K}$ and specimen voltage close to $10 \mathrm{kV}$, using neon as imaging gas on a CAMECA energy compensated tomographic atom probe, with inbuilt FIM capabilities. APT measurements were performed on a CAMECA LEAP 3000 . The specimen temperature was kept constant at $80 \mathrm{~K}$, and the pulse fraction set at $20 \%$ of the standing voltage.

SANS measurements were carried out at Institut Laue-Langevin in Grenoble, France, on beamline D22. SANS under a saturating magnetic field is classically used to characterise the non-magnetic carbides or carbo-nitrides that act like magnetic holes in the ferritic matrix (see e.g.[15-17]). It has proven to be sufficiently sensitive to quantify 
precipitation states with very low volume fractions (down to $10^{-4}$ ) [15]. The present measurements were carried out under a magnetic field of $1.5 \mathrm{~T}$. Samples had a $1 \mathrm{~cm}^{2}$ illuminated area, and a thickness of $3 \mathrm{~mm}$. A wavelength of $0.6 \mathrm{~nm}$, above the Bragg cut-off, ensured that no parasitic diffraction effects would mask the weak signal arising from precipitation. Three sample-to-detector distances were used for obtaining a range of scattering vectors from 0.1 to $4 \mathrm{~nm}^{-1}$. The nuclear and magnetic contributions to the scattering signal were separated using the beamline software GRASP, in the same way as in [15].

\section{Electron microscopy and atom probe observations}

Fig. 1(a) shows a low magnification High Resolution TEM micrograph obtained on the alloy aged $1.8 \times 10^{5} \mathrm{~s}$ at $600{ }^{\circ} \mathrm{C}$. Very fine platelets are clearly visible with a high density, lying on the $\{100\}$ matrix planes. An enlargement is shown in Fig. 1(b), where a single plane defect is clearly observed; note that this platelet is not associated with any dislocation. Fig. 1(c) shows the same material, imaged by FIM. On this micrograph, a brightly imaging plate lying on a $\{100\}$ matrix plane is observed. This bright contrast is characteristic of a phase exhibiting a higher evaporation field as compared to $\mathrm{Fe}$, consistent with the presence of an $\mathrm{NbN}$ particle, as previously shown.

The same specimen was subsequently analysed by atom probe tomography. A similar section to the one of the FIM micrograph is obtained, as shown in Fig. 2. In the presented volume of $60 \times 60 \times 300 \mathrm{~nm}^{3}$, only niobium atoms are represented in green. During the atom probe analysis, the vast majority of niobium atoms are detected as $\mathrm{NbN}$ molecular ions, and very few as single $\mathrm{Nb}$ ions. This evidences the strong cohesive energy of the $\mathrm{NbN}$ pairs (consistent with the bright contrast observed in the FIM image), and indicates that the niobium rich regions are quasi-stoichiometric $\mathrm{NbN}$ particles. The number density of niobium nitrides is clearly much higher than in the presented FIM micrograph. Several analyses were conducted, leading to similar results, indicating that such a distribution is representative of the material's nanostructure. An interesting aspect revealed by this reconstruction is that the precipitation (nucleation) mode of these nitrides is dual. The vast majority of nitrides appears to be homogeneously distributed in the material, and therefore are likely to be nucleated homogeneously. By contrast, three alignments of larger particles are also observed in these reconstructions. The reconstructed volume was oriented along three different directions, in order to reveal these alignments. Theses alignments are characteristic of particles that nucleated heterogeneously on dislocation lines. But, as expected for this type of material with a relatively low density of dislocations, heterogeneous nucleation is marginal as compared to homogeneous. In this particular specimen, about $90 \%$ of the particles are homogeneously distributed in the matrix. Although their exact morphology is difficult to determine by APT due to trajectory aberrations, one can estimate that their average diameter is of the order of $10 \mathrm{~nm}$, consistently with HREM observations (Fig. 1). Their density is of the order of $10^{22}$ to $10^{23}$ particles $/ \mathrm{m}^{3}$.

\section{SANS measurements}

The inset in Fig. 3 shows an example of a SANS image obtained on the material aged $1.8 \times 10^{5} \mathrm{~s}$ at $600{ }^{\circ} \mathrm{C}$. The classical butterfly contrast is obtained, evidencing the presence of a phase with different magnetic properties as compared to the matrix. The horizontal direction provides the nuclear contribution to the scattering intensity, while the vertical direction provides the sum of the nuclear and magnetic contributions. The two contributions can be separated using the complete detector information by using a $\sin ^{2}$ fit of the intensity $v s$. the angle with the direction of the applied magnetic field [15].

Fig. 3 shows the evolution (in a log-log plot) of the scattered intensity vs. the scattering vector for the different ageing times investigated, between $300 \mathrm{~s}$ and $1.8 \times 10^{5} \mathrm{~s}$. The nuclear intensity does not depend on the ageing time. Since a low nuclear contrast is expected from the $\mathrm{NbN}$ precipitates in $\mathrm{Fe}$, the observed nuclear signal probably arises from the presence of structural defects or a few larger particles. On the other hand, the magnetic contrast is observed to rise by an order of magnitude in the investigated ageing time scale, and can therefore safely be related to the presence of the high density of $\mathrm{NbN}$ platelets evidenced by electron microscopy and atom probe techniques.

This magnetic scattering has been modelled assuming that the platelets had an oblate ellipsoidal shape (with two equal half axis a and the third half axis $c<a$ ), with a constant thickness ( $c$ parameter) and a diameter obeying a log-normal distribution, with a relative width of the distribution of 0.5 (for details on the equations used for the modelling refer to $[15,18])$. In Fig. 3 the modelled intensity is compared with the experimental data for the 5 investigated ageing times. A good agreement is obtained throughout the ageing sequence. From this fit of the data one can obtain both the plate thickness and their average radius (half diameter in the plate plane). The evolution of these two parameters with ageing time is shown in Fig. 4. The a

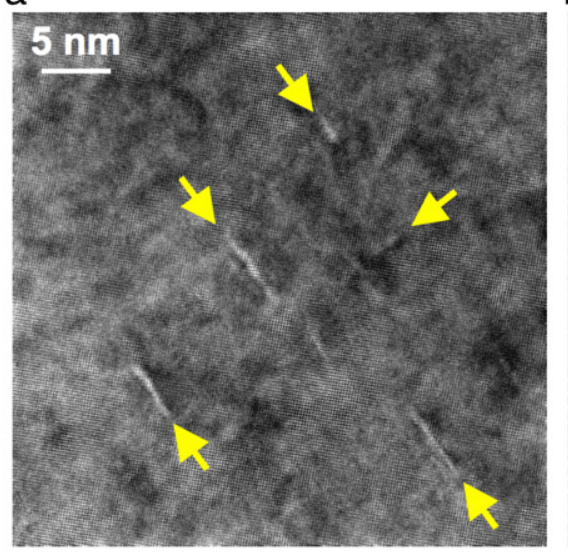

b

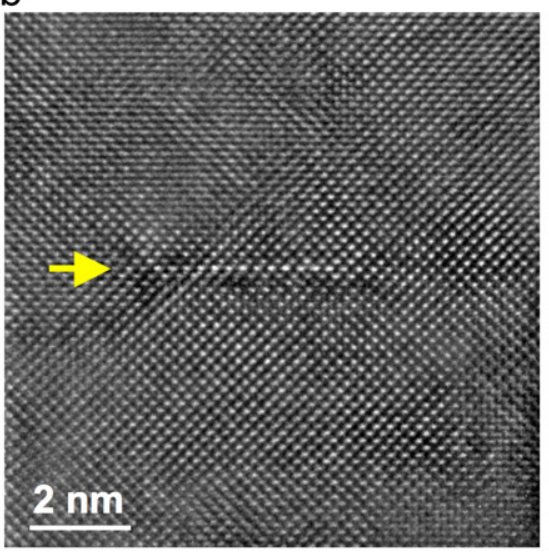

C

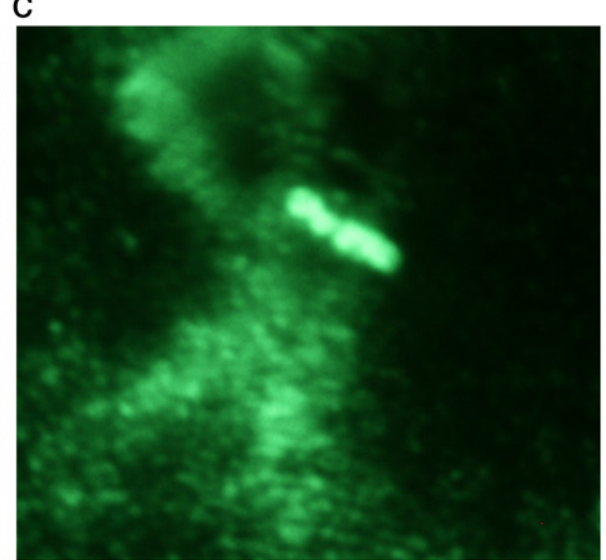

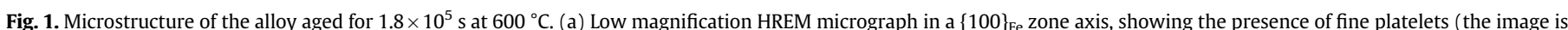

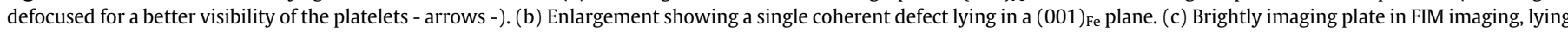

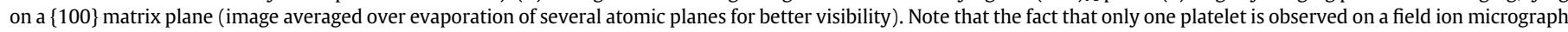
is very unlikely for this ageing condition, and not representative of the general situation. This particular micrograph was selected only for illustrative purposes. 

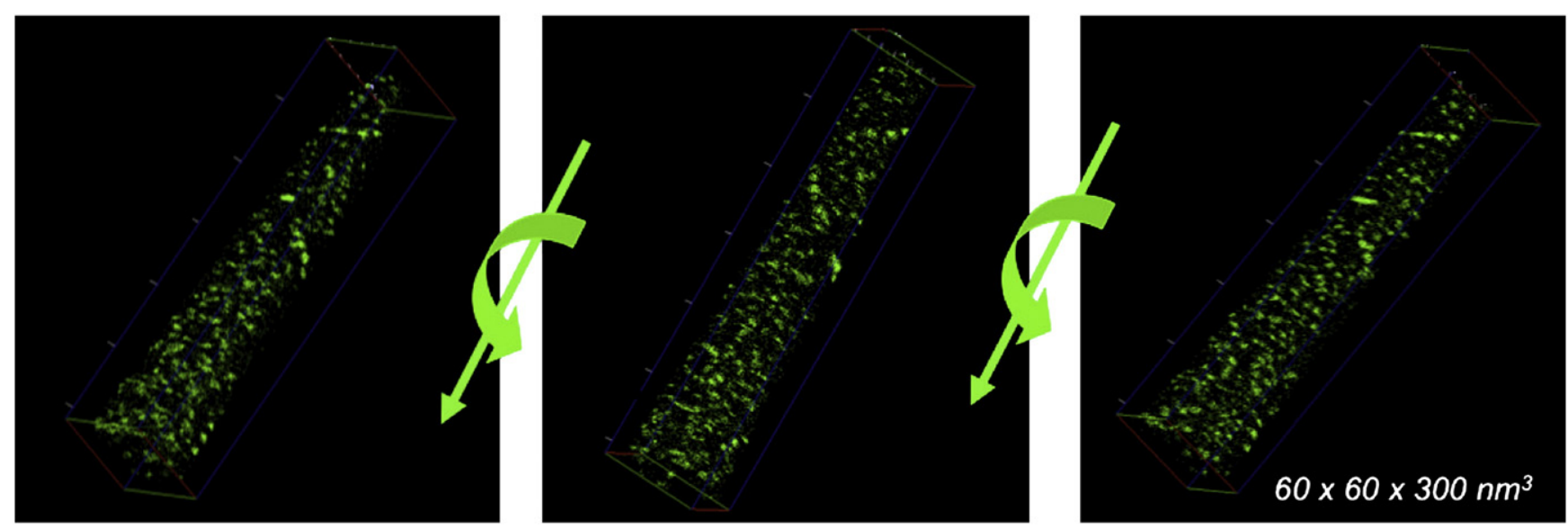

Fig. 2. APT reconstructed volume of the material aged $1.8 \times 10^{5} \mathrm{~s}$ at $600{ }^{\circ} \mathrm{C}$, showing a high density of fine platelets. Only niobium atoms are represented as green dots.

thickness $(=2 c)$ is found to be very low, below $1 \mathrm{~nm}$, consistently with what has been found in the recent literature. Moreover, this thickness is observed to be constant within the ageing time scale investigated, which means that these platelet precipitates experience in-plane growth at this temperature but no transition towards the equiaxed equilibrium $\mathrm{NbN}$ precipitates. The radius of platelets is observed to increase steadily with ageing time up to about $5 \mathrm{~nm}$ for $1.8 \times 10^{5} \mathrm{~s}$ of ageing. This measurement is in good agreement with the APT and HREM observations above.

Under the hypothesis that precipitates are magnetic holes embedded in a magnetically saturated matrix, the measurement of the scattering integrated intensity provides a measurement of precipitate volume fraction (see e.g. [15]). Moreover, dividing the volume fraction by the average particle volume $\left(4 \pi a^{2} c / 3\right)$ provides a measurement of the precipitate number density. These two measurements are shown in Fig. 4, along with the evolution of the material's hardness. The volume fraction is observed to increase steadily with ageing time in a similar fashion as the plate radius. The precipitate density is observed to be remarkably constant, except for a decrease at the last ageing time, which indicates that the temperature and ageing times studied here mainly concern the growth stage of these precipitates. It can be further inferred from the present results that nucleation of this platelet $\mathrm{NbN}$ phase occurs extremely fast, namely in the 5 first minutes at $600{ }^{\circ} \mathrm{C}$. The maximum precipitate density, of the order of $10^{22} \mathrm{~m}^{-3}$, is fully consistent with the atom probe tomography observations. The material's hardness increases in a similar manner as the particle volume fraction.

\section{Conclusions}

In the present work, we have evidenced that in a model Fe-Nb-N alloy aged at low temperature, precipitation occurred via a precursor to the equilibrium $\mathrm{NbN}$ structure that forms as extremely thin platelets less than $1 \mathrm{~nm}$ thick, lying on the $\{100\}$ matrix planes. These

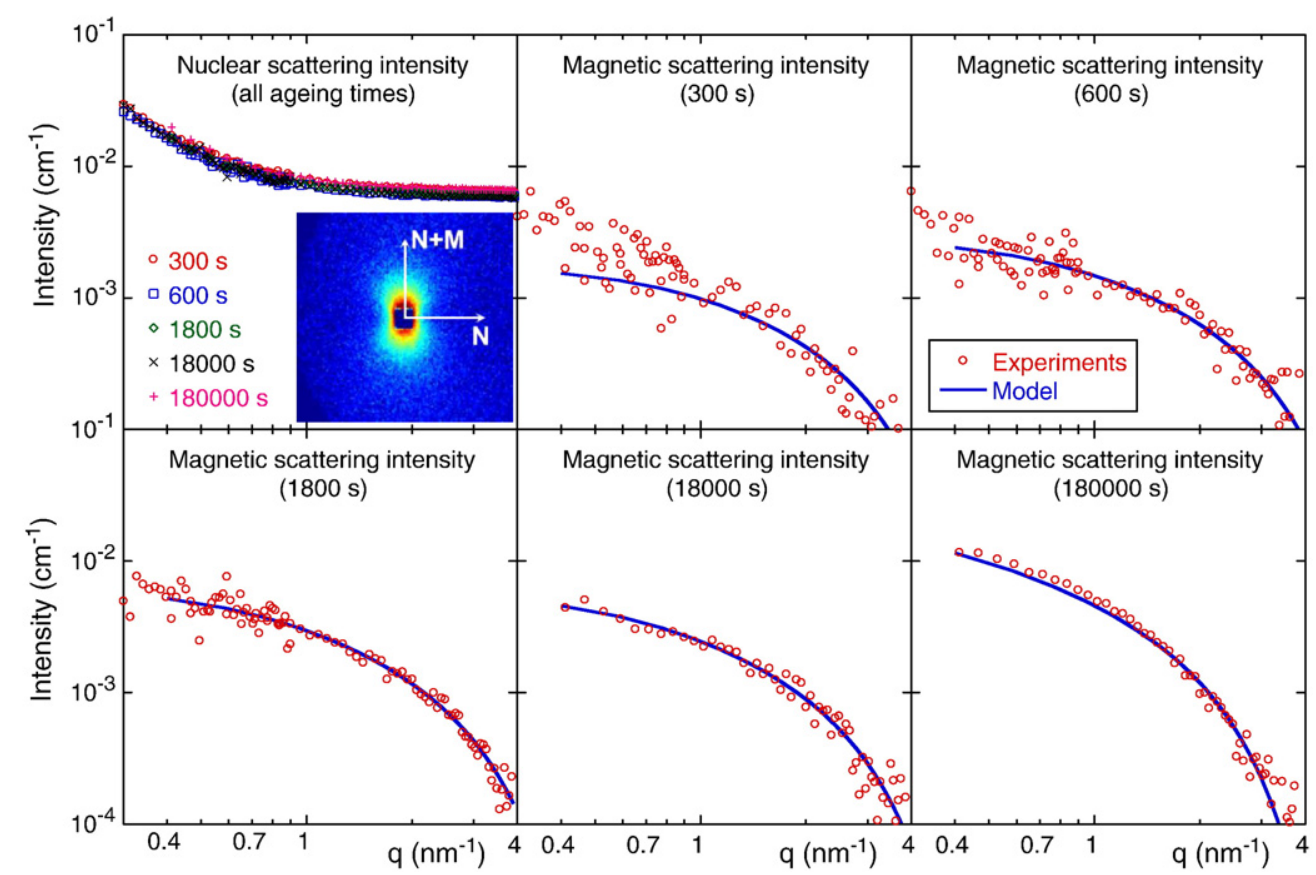

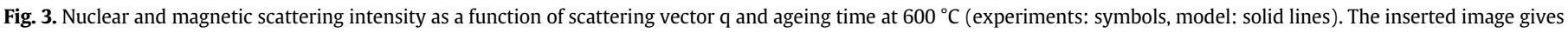

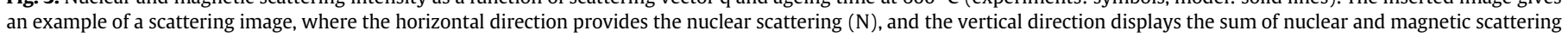
$(\mathrm{N}+\mathrm{M})$. 


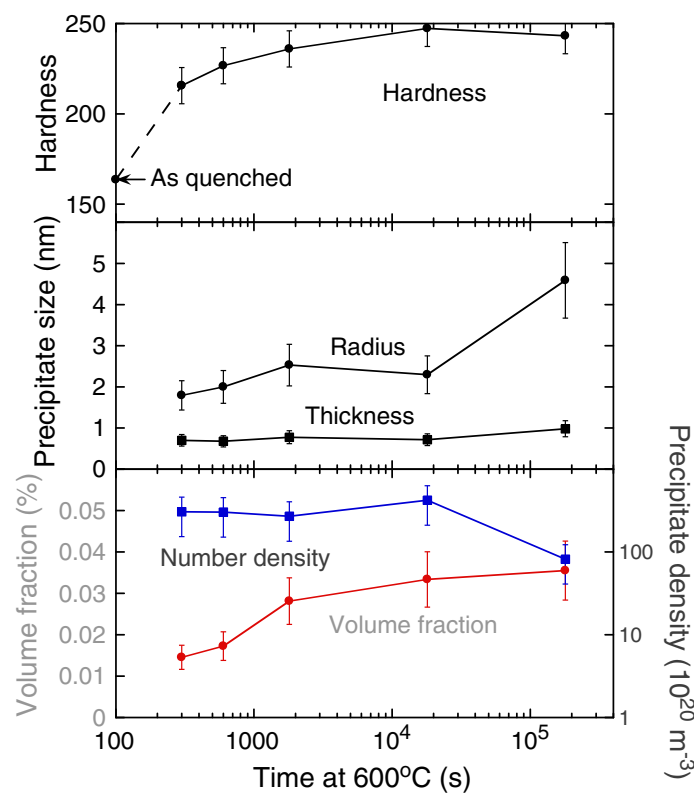

Fig. 4. Evolution of hardness, precipitate size (obtained from the model adjustments shown in Fig. 3), volume fraction and precipitate density as a function of ageing time at $600{ }^{\circ} \mathrm{C}$.

platelets nucleate very fast at the investigated temperature $\left(600^{\circ} \mathrm{C}\right)$, and in 5 min the maximum precipitate number density is achieved. They subsequently grow in plane, and in the investigated time scale no thickening or transition towards a more equiaxed morphology is observed, despite the occurrence of some precipitate coarsening for the longest ageing time.

\section{Acknowledgements}

The authors would like to acknowledge the help from Dr. Charles Dewhurst, from Institut Laue-Langevin, Grenoble, for the SANS experiments. This research was conducted in the framework of the research program 'ContraPreci' supported by the French Agence Nationale de la Recherche (contract no 06BLAN-0205). Thanks are due to the French national network METSA (www.metsa.fr) for the access to the Titan microscope at IM2NP laboratory (University of Marseille, France), where Dr. Thomas Neisius is gratefully acknowledged for his assistance.

\section{References}

[1] Perez M, Courtois E, Acevedo D, Epicier T, Maugis P. Phil Mag Lett 2007;87(9): 645-56.

[2] Courtois E, Epicier T, Scott C. Micron 2006;37(5):492-502.

[3] Wilson JA, Craven AJ. Ultramicroscopy 2003;94(3-4):197-207.

[4] Van Dijk NH, Offerman SE, Bouwman WG, Rekveldt MT, Sietsma J, Van der Zwaag S, et al. Met Mater Trans A 2002;33(7):1883-91.

[5] Palmiere EJ, Garcia CI, DeArdo AJ. Met Mater Trans A 1996;27(4):951-60.

[6] Charleux M, Poole WJ, Militzer M, Deschamps A. Met Mater Trans A 2001;32(7): $1635-47$.

[7] Rainforth WM, Black MP, Higginson RL, Palmiere EJ, Sellars CM, Prabst I, et al. Acta Mater 2002;50(4):735-47.

[8] Danoix F, Bemont E, Maugis P, Blavette D. Adv Eng Mater 2006;8(12):1202-5.

[9] Brenner SS, Goodman SR. Scripta Metall 1971;5(10):865-9.

[10] Jack DH. Acta Metall 1976;24(2):137-46.

[11] Driver JH, Papazian JM. Acta Metall 1973;21(8):1139-49.

[12] Isheim D, Seidman DN. Met Mater Trans A 2002;33(8):2317-26.

[13] Jessner P, Danoix R, Hannoyer B, Danoix F. Ultramicroscopy 2009;109(5):530-4.

[14] Tingaud D, Maugis P. Comp Mater Sci 2010;49(1):60-3.

[15] Perrard F, Deschamps A, Bley F, Donnadieu P, Maugis P.J Appl Crystallogr 2006;39(4): 473-82.

[16] Leitner H, Staron P, Clemens H, Marsoner S, Warbichler P. Mat Sci Eng A 2005;398(1-2): 323-31.

[17] Wiskel J, Ivey D, Henein H. Met Mater Trans B 2008;39(1):116-24.

[18] Deschamps A. Geuser F J Appl Cryst 2011;44:343-52. 